\title{
Congenital cutaneous candidiasis and candidemia in a preterm infant: A case report
}

\author{
Sarah Oberhauser*1, Sebastian Böhm ${ }^{1}$, Anita Niederer ${ }^{2}$, Bjarte Rogdo ${ }^{1}$ \\ ${ }^{1}$ Pediatric and Neonatal intensive care unit, Children's Hospital of Eastern Switzerland, St. Gallen, Switzerland \\ ${ }^{2}$ Department of Infectiology, Children's Hospital of Eastern Switzerland, St. Gallen, Switzerland
}

Received: October 30, 2016

DOI: $10.5430 /$ crcp.v4n1p68
Accepted: December 11, $2016 \quad$ Online Published: January 19, 2017

URL: http://dx.doi.org/10.5430/crcp.v4n1p68

\begin{abstract}
We report a case of congenital cutaneous candidiasis (CCC) progressing to candidemia in an extremely low birth weight infant. A high level of suspicion concerning candida sepsis as well as timely diagnostic work-up and treatment can be lifesaving when a preterm infant presents with lesions suggestive of congential cutaneous candidiasis.
\end{abstract}

Key Words: Candidemia, Congenital cutaneous candidiasis, Preterm infant, Fluconazole, Leukemoid reaction

\section{INTRODUCTION}

Congenital cutaneous candidiasis (CCC) is a rare condition in the neonate associated with chorioamnionitis due to Candida spp. ${ }^{[1]}$ Patients typically present on the first day of life (DOL) with skin lesions of various morphologies. A progression to systemic candida infection occurs in up to $50 \%$ of $\mathrm{CCC}$ cases in extremely low birth weight (ELBW) infants. ${ }^{[2]}$ We report a case of candidemia in an ELBW infant with congential cutaneous candidiasis. It is pointed out why a high level of suspicion concerning candida sepsis can be lifesaving when a preterm infant presents with lesions suggestive of $\mathrm{CCC}$ at birth and how antifungal treatment should be done.

\section{Case presentation}

A 17-year-old primigravida with an otherwise uncomplicated pregnancy presented at $24+6$ weeks of gestation with signs of preterm labour. She was given tocolytic therapy and antenatal steroids as well as antibiotic treatment with cefuroxime. Due to suspected chorioamnionitis and signs of foetal distress, caesarean section was performed at $25+5$ weeks of gestation. Amniotic fluid was green and foul smelling. The male infant had a birth weight of $920 \mathrm{~g}$. Apgar scores were 5, 5 and 7 at 1,5 and 10 minutes respectively. Due to respiratory distress and high oxygen requirements, he was immediately intubated and treated with surfactant. Empiric antibiotic treatment with amoxicillin and gentamicin was started after birth because of suspected neonatal infection. In the following hours, he developed tachycardia and fever. On physical examination white papules were noticed on the face, the sole of feet as well as in the axillary and inguinal regions (see Figures 1-2).

The initial white blood count revealed leucocytosis $(41 \mathrm{G} / \mathrm{L})$ and mild thrombocytopenia $(100 \mathrm{G} / \mathrm{L})$. C-reactive protein was $14 \mathrm{mg} / \mathrm{L}$. A leukemoid reaction with a maximum leucocyte count of $103 \mathrm{G} / \mathrm{L}$ developed within the first days of life. Blood glucose levels were elevated with a maximal value of $25 \mathrm{mmol} / \mathrm{L}$, so that insulin therapy was required (see Figure $3)$.

Due to persisting signs of infection and growth of $C$. albicans in amniotic fluid and maternal vaginal swabs, fluconazole was added to the antimicrobial therapy on the $2^{\text {nd }}$ DOL. The rash was fading and disappeared shortly after. Ultrasound

*Correspondence: Sarah Oberhauser, M.D.; Email: sarah.oberhauser@kispisg.ch; Address: Claudiusstrasse 6, 9000 St. Gallen, Switzerland. 
screening for systemic candidiasis the following day was negative for renal or cerebral abscess and endophthalmitis was excluded by ophthalmologic examination. Urine cultures from the $3^{r d}$ DOL showed growth of $C$. albicans 3 days later and blood culture from the $6^{\text {th }}$ DOL grew $C$. albicans after 24 hours. Culture and polymerase chain reaction (PCR) taken from cerebrospinal fluid (CSF) on the $11^{\text {th }}$ DOL were both negative for $C$. albicans. Despite systemic antimycotic treatment, the patient deteriorated with respiratory failure, requiring high frequency oscillation ventilation on the $7^{\text {th }} \mathrm{DOL}$ and blood and urine cultures still grew C. albicans, without bacterial growth. Susceptibility testing of $C$. albicans to fluconazole revealed a high minimal inhibitory concentration $(3 \mu \mathrm{g} / \mathrm{ml}),{ }^{[3]}$ and treatment was changed to liposomal amphotericin B. Because of persisting candidemia 48 hours later therapy was again changed to micafungin on the $9^{\text {th }}$ DOL. Subsequently leucocyte count and blood glucose levels normalized over the next days (see Figure 3). Blood cultures remained negative from the $12^{\text {th }}$ DOL and clinical condition improved markedly. Cerebral ultrasound and ophthalmologic follow up exams remained normal. The antifungal treatment with micafungin (10 mg/kg/day) was stopped after 3 weeks.

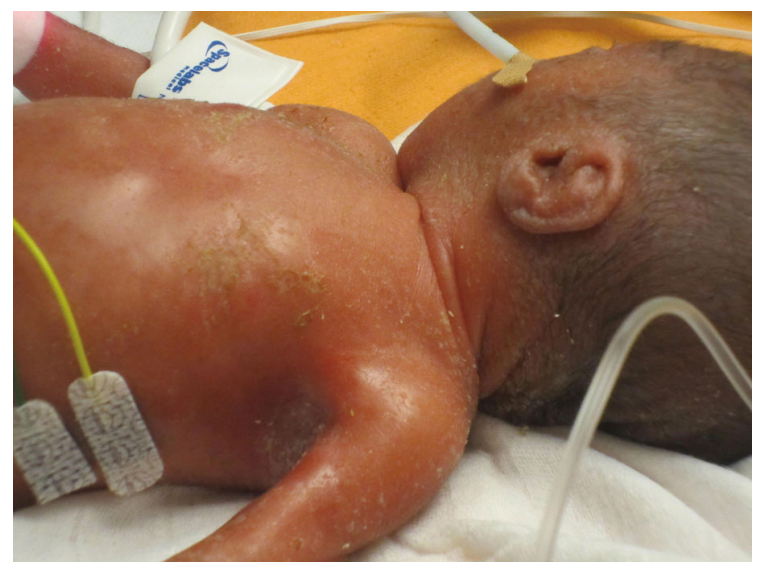

Figure 1. White papules on the face, upper trunk and axillae

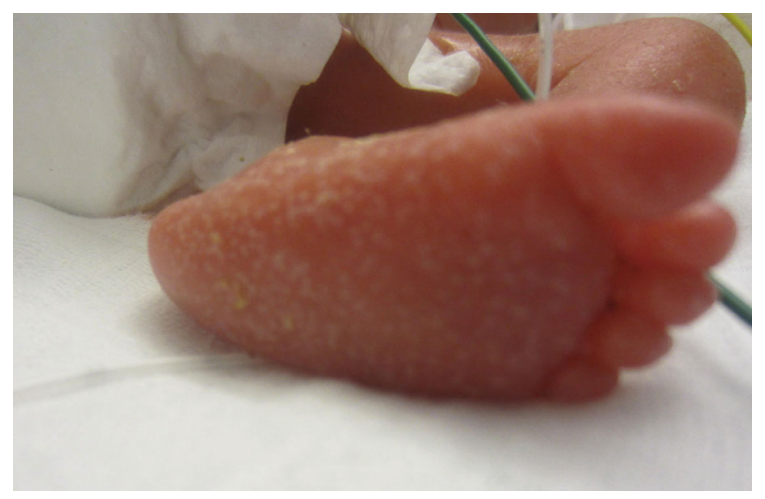

Figure 2. White papules on sole of the feet

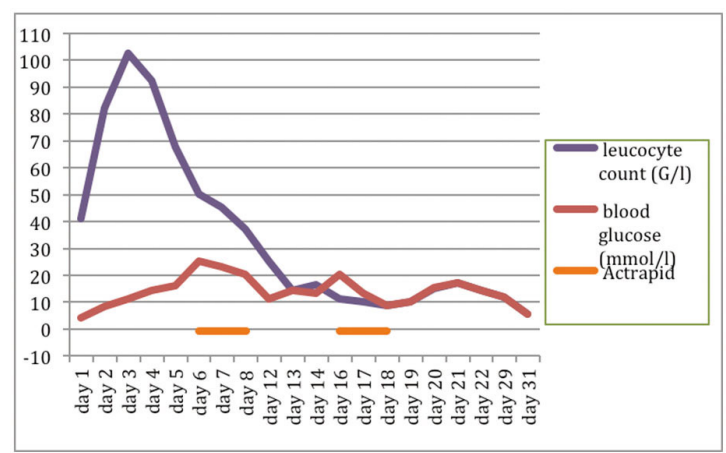

Figure 3. Leucocyte count and blood glucose levels day 1-31, and Insulin therapy

Placenta histology showed signs of acute chorioamnionitis with omphalovasculitis and funisitis with ubiquitous presence of fungal elements (see Figure 4).

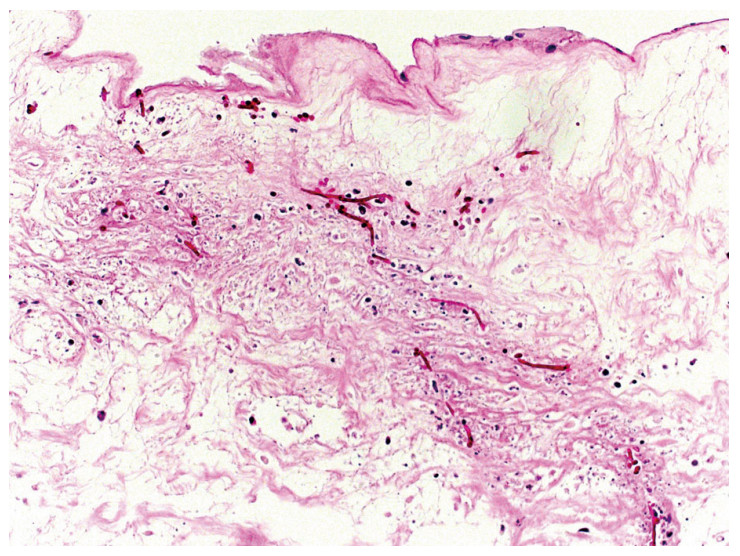

Figure 4. Acute fungal funisitis with evidence of hyphea and spores of candida species (PAS-staining, 20× augmentation)

The neonatal course was further complicated by a central catheter associated gram-negative sepsis with Serratia marcescens at 3 weeks of age, as well as bronchopulmonary dysplasia.

Despite this complicated neonatal course, neurodevelopmental examination at 3 months of corrected age revealed normal motor and mental development. Later, at the corrected age of 23 months the patient showed a delay of mental development appropriate to a child of 16 months and the linguistic competences were appropriate to a child of 13 to 15 months. Motor skills were within normal ranges for corrected age.

\section{Discussion}

C. albicans is a common vaginal pathogen found in up to $30 \%$ of pregnant women, however Candida is recovered by culture from less than $1 \%$ of placentas. ${ }^{[2,4,5]}$ Known risk factors for $\mathrm{CCC}$ are intrauterine devices (IUD) during early pregnancy 
and cervical cerclage later in pregnancy. ${ }^{[2]}$ Ascending infection may occur either with rupture of membranes or through intact membranes resulting in whitish plaques on membranes and umbilical cord along with skin lesions. ${ }^{[6]}$ Due to immaturity of the immune system and of the mucocutaneous barrier, ELBW infants are at substantially higher risk for systemic involvement than term infants. Some authors propose to consider CCC in preterm infants as an invasive Candida infection and to start systemic treatment as soon as diagnosis is suspected. ${ }^{[7]}$

In our case, placenta histology showed acute fungal chorioamnionitis, confirming the suspected diagnosis. There was no history of IUD, cervical cerclage or premature rupture of membranes. The infant had multiple skin lesions at birth as well as clinical signs and changes in white blood count consistent with systemic infection. Therefore, antifungal treatment was started on $2^{\text {nd }} \mathrm{DOL}$, when cultures of amniotic fluid grew $C$. albicans. Routine antifungal prophylaxis is not given in our centre, owing to very low rates of neonatal fungal infection.

Neonates with clinical signs of systemic fungal disease and cutaneous lesions at birth should undergo a complete workup for systemic fungal disease, including renal, ophthalmologic and central nervous system evaluation. ${ }^{[1]}$ In our patient $C$. albicans grew in blood and urine, but no other organ system was involved. Observed hyperglycaemia can be interpreted as a marker for invasive fungal infection. ${ }^{[8]}$

Retrospectively our patient could possibly have benefited from a higher dosing regimen of fluconazole, especially in view of the high MIC of $C$. albicans for fluconazole. Cur- rently recommended dosing regimens for invasive candida infection are considerably higher than previously used regimens $\left(6-12 \mathrm{mg} / \mathrm{kg}\right.$ every 72 hours) ${ }^{[9]}$ Wade et al. show that a therapeutic concentration of fluconazole in premature infants with invasive candidiasis requires substantially higher dosing of $12 \mathrm{mg} / \mathrm{kg}$ every 24 hours, ${ }^{[10]}$ and loading dose of $25 \mathrm{mg} / \mathrm{kg}$ is recommended to achieve therapeutic levels faster. ${ }^{[9,10]}$

Invasive candida infections in ELBW infants are associated with high mortality rates and increased risk for poor neurodevelopmental outcome. ${ }^{[11]}$ In our patient CCC rapidly progressed to candida sepsis. After changing antifungal therapy to micafungin, the clinical situation improved rapidly. At 23 months of corrected age, the patient showed moderate delays in cognitive development, with motor skills within normal range for age. Although the extreme prematurity must be considered the main risk factor for development delays, the two episodes of sepsis contributed to a higher risk of neurodevelopmental delays in our patient.

\section{ACKNOWLEDGEMENTS}

Regulo Rodriguez, senior consultant, Institute of Pathology, Kantonsspital St. Gallen, for the appropriation of the histopathological pictures. Christian Kahlert, senior consultant, Department of Infectiology, Children's Hospital St. Gallen, for his participation at this article. Michael von Rhein, senior consultant, Department of Pediatric Development, Kantonsspital Winterthur, for the disclosure of the follow-up examination.

\section{CONFLicts OF InTEREST Disclosure}

The authors declare no conflicts of interest.

\section{REFERENCES}

[1] Barton M, O'Brien K, Robinson JL, et al. Invasive candidiasis in low birth weight preterm infants: risk factors, clinical course and outcome in a prospective multicenter study of cases and their matched controls. BMC Infect Dis. 2014; 14: 327. PMid: 24924877. https://doi.org/10.1186/1471-2334-14-327

[2] Darmstadt GL, Dinulos JG, Miller Z. Congenital cutaneous candidiasis: clinical presentation, pathogenesis, and management guidelines. Pediatrics. 2000; 105(2): 438-44. PMid: 10654973. https: //doi.org/10.1542/peds.105.2.438

[3] Eucast: Fluconazole - Rationale for the EUCAST clinical breakpoints, Ver. 2.0 [Internet]. Available from: http://www. eucast.o rg/fileadmin/src/media/PDFs/EUCAST_files/Rationale _documents/Fluconazole_rationale_2_0_20130223.pdf

[4] Leli C, Mencacci A, Meucci M, et al. Association of pregnancy and Candida vaginal colonization in women with or without symptoms of vulvovaginitis. Minerva Ginecol. 2013; 65(3): 303-9. PMid: 23689173.

[5] Tiraboschi ICN, Niveyro C, Mandarano AM, et al. Congenital candidiasis: confirmation of mother-neonate transmission using molecular analysis techniques. Med Mycol. 2010; 48(1): 177-81. PMid: 19306215. https://doi.org/10.3109/13693780902824962
[6] Diana A, Epiney M, Ecoffey M, et al. "White dots on the placenta and red dots on the baby": congential cutaneous candidiasis-a rare disease of the neonate. Acta Paediatr Oslo Nor 1992. 2004; 93(7): 996-9.

[7] Chen WY, Chen SJ, Tsai SF, et al. Congenital Systemic Fungus Infection in Twin Prematurity-A Case Report and Literature Review. AJP Rep. 2015; 5(1): 46-50. PMid: 26199798. https: //doi.org/10.1055/s-0035-1548730

[8] Manzoni P, Castagnola E, Mostert M, et al. Hyperglycaemia as a possible marker of invasive fungal infection in preterm neonates. Acta Paediatr Oslo Nor 1992. 2006; 95(4): 486-93.

[9] Ainsworth SB. Neonatal Formulary: Drug Use in Pregnancy and the First Year of Life. John Wiley \& Sons. 2014. 647p. https : //doi.org/10.1002/9781118819494

[10] Wade KC, Benjamin DK, Kaufman DA, et al. Fluconazole Dosing for the Prevention or Treatment of Invasive Candidiasis in Young Infants. Pediatr Infect Dis J. 2009; 28(8): 717-23. PMid: 19593252. https ://doi.org/10.1097/INF.0b013e31819f 1 f50

[11] Adams-Chapman I, Bann CM, Das A, et al. Neurodevelopmental Outcome of Extremely Low Birth Weight Infants with Candida Infection. J Pediatr. 2013; 163(4): 961-7. PMid: 23726546. https://doi.org/10.1016/j.jpeds.2013.04.034

ISSN 2331-2726 E-ISSN 2331-2734 